Benchmark. Slides were scanned and counted for the number of tumor and immune cells expressing PD-L1 in 3 parts in each slide by one pathologist. Tumor cell proportion score (TC) and immune cell proportion score (IC) were calculated.

Results: Median of TC in 22C3, SP263, and SP142 was 35.0\%, $50.7 \%$, and $9.1 \%$, respectively. Median of IC in 22C3, SP263, and SP142 was $1.7 \%, 1.8 \%$, and $1.8 \%$, respectively. Those 3 antibodies showed a high correlation in TC of PD-L1 staining. The percentage agreement was $85.7 \%$ between $22 \mathrm{C}$ and SP263 $(\kappa=0.440, \mathrm{p}=0.047)$. The percentage agreement was $92.9 \%$ and $85.7 \%$ between $22 \mathrm{C} 3$ and SP142 using $1 \%(\kappa=0.811, \mathrm{p}=0.002)$ of TC, respectively. Using clinically relevant cut-off, the percentage agreement between SP263 and 22C3, and 22C3 and SP142 was $71.4 \%$ ( $\kappa=0.152, \mathrm{p}=0.571)$ and $92.9 \%$ ( $\kappa=0.811, \mathrm{p}=0.002)$, respectively. Intratumoral agreement of positivity of PD-L1 expression among different 3 parts in the same slide was $100 \%$ in 22C3 and SP263.

Conclusion: Those 3 antibodies showed a good correlation in TC of PD-L1 expression. 22C3 and SP142 may be

interchangeable to determine PD-L1 due to its high agreement to evaluate the positivity for PD-L1 in PSTT/ETT. SP263 showed a higher positivity rate than others, and the agreement was acceptable with $22 \mathrm{C} 3$.

Oral (OMi3)

Endometrial Hyperplasia, Endometrial Intra-epithelial Neoplasia, and Endometrial Cancer

https://doi.org/10.3802/jgo.2021.32.S1.0Mi3

\section{Feasibility of identifying circulating tumor DNA based on cervical smear samples in endometrial cancer patients}

\author{
Yoo-Na Kim, ${ }^{1}$ Namsoo Kim, ${ }^{2}$ Jung-Yun Lee, ${ }^{1, *}$ Seung-Tae Lee, ${ }^{2}$ \\ Jong Rak Choi, ${ }^{2}$ Sunghoon Kim ${ }^{1}$ \\ 'Department of Obstetrics and Gynecology, Yonsei University College of \\ Medicine, Seoul, Korea (jungyunlee@yuhs.ac) \\ ${ }^{2}$ Department of Laboratory Medicine, Yonsei University College of Medicine, \\ Seoul, Korea
}

Objective: Cervical smear samples are easy to obtain and may effectively reflect the tumor environment in the pelvis due to anatomic proximity. Thus, we have investigated the feasibility of using cervical smear samples for tumor DNA analysis in endometrial cancer patients.

Methods: Patients undergoing endometrial cancer staging were prospectively enrolled since January 2021. Cervical smear samples were obtained preoperatively via vaginal sampling. Cell-free DNA was extracted and analyzed with a custom panel which covers 101 endometrial cancer-related genes. Prepared libraries were sequenced using NovaSeq 6000 System (Illumina) and analyzed using Piseq analysis (Dxome).

Results: Cervical smear samples were obtained from 13 patients, and among them whole blood was available for ctDNA analysis in 12 patients. This predominantly early-stage cohort consisted of stage IA ( $n=7)$, stage IB ( $n=3)$, and stage II, III, and IV patients ( $\mathrm{n}=1$ for each). Overall, the detection rate for cervical cytology-based tumor DNA was 8 out of 13 patients $(62 \%)$, whereas only 2 out of 13 patients $(15 \%)$ had detectable ctDNA based on blood. Specifically, for stage IA patients, ctDNA was detected in 3 out of 7 patients, despite that all 7 patients had normal PAP smear result. Based on cytology-based tumor DNA analysis of cervical smear samples, the most frequently identified gene mutations were PTEN ( $n=6)$, PIK3CA ( $n=4)$, ARID1A (n=3), KRAS (n=3), TP53 (n=2), MSH6 (n=2), and ATM $(n=2)$.

Conclusion: In this pilot study with predominantly early-stage endometrial cancer patients, cervical smear was more sensitive than whole blood or conventional PAP smear in terms of detecting tumor DNA.

Oral (OMi4)

Gynecologic Cancer Screening

https://doi.org/10.3802/jgo.2021.32.S1.0Mi4

\section{Pilot study on biomarkers for screening endometrial hyperplasia and early-stage endometrioid cancer}

\author{
Wei Li Lin,' Chao-Min Cheng, ${ }^{2}$ Ting-Chang Chang ${ }^{*}$ \\ 'Department of Obstetrics and Gynecology, Chang Gung Memorial \\ Hospital (Linkou), Tao-Yuan, Taiwan (tinchang.chang@gmail.com) \\ ${ }^{2}$ Institute of Biomedical Engineering, National Tsing Hua University, Hsinchu, \\ Taiwan
}

Objective: Endometrial cancer is the most common malignancy of the female reproductive system in developed countries including Taiwan. Ways to screen precancerous endometrial lesion and early-stage endometrial cancer are mandatory. We performed proteomics study on endometrioid endometrial cancer and proteinase-3 (PRTN-3) showed significant expression in cancerous tissue compared with normal endometrial tissue. High expression of vascular endothelial growth factor (VEGF) and interleukin-6 (IL-6) was noted through literature review. We then conducted a pilot study to check if these are effective markers in vaginal discharge for the detection of early-stage endometrial cancer and its precancerous lesion.

Methods: After granted by Institutional Review Board, vaginal discharge samples of 133 patients between 2015 and 2017 at 
Linkou Chang Gung Memorial Hospital were collected. Study subjects were those: 1 ) with known endometrial lesions, which including any type of endometrial hyperplasia and any stage of endometrioid carcinoma of the endometrium, 2) with symptoms/signs of endometrial hyperplasia or endometrial cancer, including abnormal uterine bleeding with thick endometrial stripe or medically uncontrollable uterine bleeding, as study group. Patients with benign uterine/ovarian tumors were invited as the control group. The sample was collected using a designed cotton swab by study physician and was stored in ?? $0^{\circ} \mathrm{C}$ until ELISA analysis.

Results: Statistically significant elevation of IL-6 titer and VEGF titer but not PRTN-3 among endometrial cancer patients, compared with those with normal uterus or with benign tumors, were noted. No significant differences were observed for PRTN-3 level within the groups. Algorithm for endometrial hyperplasia and endometrial cancer screening is established accordingly.

Conclusion: This study represents the first evaluation of IL-6, PRTN-3 and VEGF level extracting from vaginal fluid as potential markers in screening for endometrial cancer. As a noninvasive measure, further validation study is needed.

Oral (OMi5)

Gynecologic Pathology, Genetics and Epidemiology

https://doi.org/10.3802/jgo.2021.32.S1.0Mi5

\section{Association between hospital treatment volume and survival of surgical cases with gynecologic malignancy in Japan}

\section{Hiroko Machida, ${ }^{1}$ Koji Matsuo, ${ }^{2}$ Daisuke Aoki, ${ }^{3}$ Takayuki Enomoto, ${ }^{4}$ Aikou Okamoto, ${ }^{5}$ Hidetaka Katabuchi, ${ }^{6}$ Satoru Nagase, ${ }^{7}$ Masaki Mandai, ${ }^{8}$ Nobuo Yaegashi, ${ }^{9}$ Mikio Mikami ${ }^{1, *}$}

'Tokai University School of Medicine, Isehara, Japan (mmikami@is.icc.utokai.ac.jp)

${ }^{2}$ University of Southern California, Los Angeles, CA, USA

${ }^{3}$ Keio University School of Medicine, Tokyo, Japan

${ }^{4}$ Niigata University School of Medicine, Niigata, Japan

${ }^{5}$ Jikei University School of Medicine, Tokyo, Japan

${ }^{6}$ Kumamoto University, Kumamoto, Japan

${ }^{7}$ Yamagata University Faculty of Medicine, Yamagata, Japan

${ }^{8}$ Kyoto University Graduate School of Medicine, Kyoto, Japan

${ }^{9}$ Tohoku University, Sendai, Japan

Objective: Associations between hospital treatment volume and survival outcomes for surgical cases with 3 types of gynecologic malignancies, and the trends and contributing factors for highvolume centers were examined.

Methods: The Japan Society of Obstetrics and Gynecology tumor registry data based retrospective study examined 167,642 women with 76,255, 41,187, and 45,200 of endometrial, cervical, and ovarian tumor, respectively, who underwent primary surgery in Japan between 2004 and 2015. Associations between the annual treatment volume and overall survival (OS) for each tumor type were examined. Institutions were categorized into 3 groups (low-, moderate-, and high-volume centers) based on prior study.

Results: Among the high-volume centers, the median surgical treatment volumes were 67 (interquartile range [IQR], 59-82), 68 (IQR, 59-91), and 35 (IQR, 31-41) cases/year for endometrial, cervical, and ovarian tumors, respectively. On multivariate analysis, younger age, rare tumor histology, and lymphadenectomy were contributing factors for women at high-volume centers (all, $\mathrm{p}<0.001$ ). During the study period, the proportion of high-volume center treatments was decreased, whereas that of low-volume center treatments was increased for the 3 tumor types (all, $\mathrm{p}<0.001$ ). Surgical treatment at high-volume centers improved OS than that at other centers (adjusted-hazard ratio [95\% confidence interval]: 0.82 [0.760.88 ], 0.83 [0.76-0.91], and 0.88 [0.84-0.93] for endometrial, cervical, and ovarian tumors, all, $\mathrm{p}<0.001)$.

Conclusion: For women with gynecologic malignancy in Japan, surgical treatments at high-volume centers conferred survival benefits. The practice pattern shifted with scattering of patients and treatments at high-volume centers were decreasing.

Oral (OMi6)

Gynecologic Pathology, Genetics and Epidemiology

https://doi.org/10.3802/jgo.2021.32.S1.0Mi6

\section{Mutations of DNA damage repair pathway- related genes in ovarian caner detected by next-generation sequencing}

\author{
Rong Feng, Shoutai Ding, Huiling Chen, Chaoran Xia, \\ Zhixiang Yan, Peng Luo, Jiajia Xu" \\ Topgen Biological Medicine Technology Co., Ltd., Shanghai, China \\ (xujiajia@topgen.com.cn)
}

Objective: To study the mutations occurred in related genes in DNA damage repair pathway (DDR) in ovarian cancer (OC) patients detected by next-generation sequencing (NGS). Methods: After NGS of tissue samples from 205 ovarian cancer cases, the mutation types and mutation frequencies of DDR pathway-related genes were classified and analyzed. Two-hundred five female Chinese patients with OC (including primary peritoneal cancer and fallopian tube cancer) were involved and detected by OncoDrug-Seq ${ }^{\mathrm{TM}} 603$-gene panel assay through NGS using Illumina NovaSeq 6000.

Results: The mutated genes in the 205 OC patients were 